\title{
Achat d'antibiotiques par les hôpitaux canadiens, 2007-2011
}

\author{
Finley $\mathbf{R}^{1^{*}}$ \\ ${ }^{1}$ Centre des maladies infectieuses d'origine alimentaire, environnementale et zoonotique, Agence de la santé \\ publique du Canada, Guelph (Ontario) \\ * Correspondance à : rita.finley@phac-aspc.gc.ca
}

\section{Résumé}

Objectif : Décrire les profils d'achat d'antibiotiques par les hôpitaux de cinq régions du Canada entre 2007 et 2011.

Méthodologie : L'ensemble de données du Canadian Drugstore and Hospital Purchases Audit (CDH) mesure la valeur en dollars et le volume unitaire des produits pharmaceutiques de plus de 700 hôpitaux et permet, par extrapolation, de déterminer les achats de plus de 800 hôpitaux au Canada. Les données sur la population générale ont été utilisées pour déterminer les taux d'achat en regard principalement du volume unitaire.

Résultats : En 2011, les achats totaux d'antibiotiques par les hôpitaux canadiens ont augmenté de $7 \%$ par rapport à 2010, de légères augmentations ayant été observées au niveau des achats d'antibiotiques oraux (hausse de $8 \%$ ) et parentéraux (hausse de $3 \%$ ). Les antibiotiques considérés comme étant de très haute importance en médecine humaine (catégorie I) ont continué de représenter un pourcentage élevé de la totalité des antibiotiques utilisés dans les hôpitaux en 2011. Dans l'ensemble, c'est au Manitoba que la consommation d'antibiotiques a été la plus élevée, celle-ci s'établissant à 2,61 doses thérapeutiques quotidiennes par 1000 habitants-jour (DTQ/1 000 habitants-jour), et en Ontario qu'elle a été la plus faible (1,26 DTQ/1 000 habitants-jour). En 2011, la plus forte consommation d'antibiotiques de catégorie I (43\%; 0,62/1,43 DTQ/1 000 habitants-jour) a été enregistrée au Nouveau-Brunswick, un record attribuable à l'augmentation de la consommation de fluoroquinolones dans cette province.

Conclusion : Les hôpitaux canadiens achètent davantage d'antibiotiques et ont légèrement augmenté leur consommation d'antibiotiques oraux et parentéraux. Dans l'ensemble, c'est au Manitoba que la consommation a été la plus forte et en Colombie-Britannique que le coût total a été le plus élevé. À l'opposé, l'Ontario a présenté le plus faible taux de consommation d'antibiotiques et le coût global le plus bas.

\section{Introduction}

Le Programme intégré canadien de surveillance de la résistance aux antimicrobiens (PICRA) surveille les tendances en matière de résistance aux antimicrobiens et d'utilisation d'antimicrobiens chez certains organismes bactériens isolés de sources humaines, animales et alimentaires de partout au Canada. Le programme est fondé sur plusieurs composantes de surveillance représentatives et méthodologiquement unifiées qui peuvent être liées pour examiner la relation entre les antibiotiques utilisés chez les animaux destinés à la consommation et les humains et évaluer leurs répercussions subséquentes sur la santé. Ces renseignements permettent (i) la création de politiques basées sur des données probantes afin de contrôler l'utilisation des antimicrobiens dans les hôpitaux, au sein de la communauté et dans les milieux agricoles, permettant ainsi de prolonger l'efficacité de ces médicaments, ainsi que (ii) l'identification de mesures appropriées pour freiner l'apparition de bactéries résistantes et leur propagation entre les animaux, les aliments et les humains au Canada.

Les rapports de surveillance de l'utilisation des antimicrobiens chez les humains sont publiés sur une base régulière, et ce, afin de fournir un aperçu national annuel des pratiques actuelles en matière 
d'utilisation des antibiotiques au Canada, d'établir des comparaisons et de favoriser la gestion des antibiotiques et leur inscription aux listes de médicaments. L'objectif du présent document est de décrire les profils d'achat d'antibiotiques des hôpitaux de cinq régions du Canada entre 2007 et 2011.

L'information qui y est présentée est basée sur le plus récent Rapport du Programme intégré canadien de surveillance de la résistance aux antimicrobiens (PICRA) qui est accessible en ligne (1).

\section{Méthodologie}

L'Agence de la santé publique du Canada achète l'ensemble de données du Canadian Drugstore and Hospital Purchases Audit (CDH) auprès d'IMS Health Canada Inc. Cet ensemble de données mesure la valeur en dollars et le volume unitaire des produits pharmaceutiques achetés par la quasi-totalité des hôpitaux du Canada, à l'exclusion de ceux du Yukon, des Territoires du Nord-Ouest et du Nunavut. Des renseignements ont été recueillis auprès de plus de 700 hôpitaux et ont été extrapolés pour déterminer les achats de plus de 800 hôpitaux au Canada. Les provinces de l'Île-du-Prince-Édouard et de TerreNeuve-et-Labrador ont été regroupées en raison du faible volume d'achats faits par les hôpitaux de chacune de ces provinces. Comme le nombre de jours d'hospitalisation et de lits d'hôpitaux n'était pas disponible pour ces données, les renseignements sur la population générale ont été utilisés pour déterminer les taux d'achat. Dans le présent document, le terme « consommation » est utilisé pour représenter les achats effectués par les hôpitaux.

\section{Résultats}

De 2001 à 2011, les achats totaux d'antibiotiques ont augmenté de $27 \%$, passant de $34958,30 \mathrm{~kg}$ à $44406,35 \mathrm{~kg}$. Depuis 2001, toutefois, le nombre total de doses thérapeutiques quotidiennes (DTQ) par 1000 habitants-jour est demeuré relativement stable, et ce, autant pour les antibiotiques oraux que parentéraux (tableaux 1 et 2). De 2010 à 2011, de légères augmentations ont été observées pour certains antibiotiques oraux ( $8 \%$ ) et parentéraux $(3 \%)$, ces hausses étant attribuables à une légère augmentation de la consommation des tétracyclines $(25 \%)$, des associations de pénicillines, incluant les inhibiteurs de $\beta$-lactamases (25\%), et des macrolides (8\%) (Tableau 1). 
TABLEAU 1. Nombre de doses thérapeutiques quotidiennes par 1000 habitants-jour d'antibiotiques oraux achetés par les hôpitaux au Canada, de 2001 à 2011

\begin{tabular}{|c|c|c|c|c|c|c|c|c|c|c|c|c|}
\hline & \multirow{2}{*}{ Classe ATC $^{1}$ et antimicrobien } & \multicolumn{11}{|c|}{ DTQ²/1000 habitants-jours } \\
\hline & & 2001 & 2002 & 2003 & 2004 & 2005 & 2006 & 2007 & 2008 & 2009 & 2010 & 2011 \\
\hline \multirow{8}{*}{$\beta^{\beta}$} & $\begin{array}{l}\text { Association de pénicillines, incluant les inhibite urs } \\
\text { de } \beta \text {-lactamases (J01CR) }\end{array}$ & & & & & & & & & & & \\
\hline & Amoxicilline et inhibiteur enzymatique & 0,02 & 0,02 & 0,03 & 0,03 & 0,04 & 0,04 & 0,03 & 0,03 & 0,04 & 0,04 & 0,05 \\
\hline & Céphalosporines de troisième génération (J01DD) & & & & & & & & & & & \\
\hline & Céfixime & 0,01 & 0,01 & 0,01 & 0,01 & 0,01 & 0,01 & 0,01 & 0,01 & 0,01 & 0,01 & 0,01 \\
\hline & Fluoroquinolones (J01MA) & & & & & & & & & & & \\
\hline & $\begin{array}{l}\text { Ofloxacine, ciprofloxacine, norfloxacine, lévofloxacine, } \\
\text { moxifloxacine, gatifloxacine, gémifloxacine }\end{array}$ & 0,22 & 0,22 & 0,24 & 0,24 & 0,23 & 0,23 & 0,23 & 0,22 & 0,23 & 0,20 & 0,20 \\
\hline & Im idazole (J01XD) & & & & & & & & & & & \\
\hline & Métronidazole & 0,04 & 0,03 & 0,04 & 0,04 & 0,04 & 0,04 & 0,04 & 0,04 & 0,03 & 0,03 & 0,03 \\
\hline \multirow{14}{*}{ ॥ } & $\begin{array}{l}\text { Pénicillines à large spectre (J01CA) } \\
\text { Ampicilline, amoxicilline, pivampicilline, bacampicilline, } \\
\text { pivmécillinam }\end{array}$ & 0,11 & 0,11 & 0,10 & 0,09 & 0,10 & 0,09 & 0,08 & 0,08 & 0,08 & 0,09 & 0,09 \\
\hline & Pénicillines sensibles aux $\beta$-lactam ases (J01CE) & & & & & & & & & & & \\
\hline & Pénicilline $G$, pénicilline $V$ & 0,02 & 0,03 & 0,03 & 0,02 & 0,02 & 0,02 & 0,01 & 0,01 & 0,01 & 0,01 & 0,02 \\
\hline & Pénicillines résistantes aux $\beta$-lactamases (J01CF) & & & & & & & & & & & \\
\hline & Cloxacilline & 0,03 & 0,02 & 0,02 & 0,02 & 0,02 & 0,02 & 0,01 & 0,01 & 0,01 & 0,01 & 0,01 \\
\hline & Céphalos porines de première génération (J01DB) & & & & & & & & & & & \\
\hline & Céfalexine, céfadroxil & 0,05 & 0,05 & 0,05 & 0,05 & 0,06 & 0,07 & 0,05 & 0,05 & 0,05 & 0,04 & 0,05 \\
\hline & Céphalosporines de deuxième génération (J01DC) & & & & & & & & & & & \\
\hline & Céfaclor, cefprozil, céfuroxime axétil & 0,07 & 0,05 & 0,05 & 0,05 & 0,04 & 0,04 & 0,04 & 0,04 & 0,04 & 0,03 & 0,04 \\
\hline & $\begin{array}{l}\text { Association de sulfamides et de triméthoprime, } \\
\text { incluant leurs dérivés (J01E) }\end{array}$ & & & & & & & & & & & \\
\hline & $\begin{array}{l}\text { Sulfaméthoxazole et triméthoprime, sulfadiazine et } \\
\text { triméthoprime }\end{array}$ & 0,09 & 0,07 & 0,08 & 0,07 & 0,07 & 0,07 & 0,07 & 0,06 & 0,06 & 0,06 & 0,06 \\
\hline & $\begin{array}{l}\text { Macrolides (J01FA) } \\
\text { Azithromycine, clarithromycine, érythromycine, } \\
\text { spiramycine, télithromycine }\end{array}$ & 0,13 & 0,13 & 0,14 & 0,12 & 0,13 & 0,12 & 0,13 & 0,13 & 0,14 & 0,12 & 0,13 \\
\hline & Lincosamides (J01FF) & & & & & & & & & & & \\
\hline & Clindamycine & 0,01 & 0,01 & 0,01 & 0,01 & 0,01 & 0,01 & 0,02 & 0,02 & 0,02 & 0,01 & 0,01 \\
\hline \multirow{4}{*}{ III } & Tétracyclines (J01AA) & & & & & & & & & & & \\
\hline & Doxycycline, minocycline, tétracycline, déméclocycline & 0,06 & 0,05 & 0,05 & 0,06 & 0,05 & 0,06 & 0,08 & 0,12 & 0,08 & 0,08 & 0,10 \\
\hline & Dérivés du nitrofurane (J01X日) & & & & & & & & & & & \\
\hline & Nitrofurantoïne & 0,02 & 0,03 & 0,03 & 0,03 & 0,03 & 0,03 & 0,03 & 0,03 & 0,03 & 0,03 & 0,03 \\
\hline & Total (J01) & 0,88 & 0,84 & 0,87 & 0,86 & 0,86 & 0,86 & 0,83 & 0,85 & 0,84 & 0,78 & 0,84 \\
\hline
\end{tabular}

${ }^{1}$ ATC $=$ Système de classification anatomique, thérapeutique et chimique

${ }^{2} \mathrm{DTQ}=$ Dose thérapeutique quotidienne

${ }^{3}$ Les chiffres romains de I à III indiquent l'importance des antibiotiques en médecine humaine, selon les catégories du système de classification établi par la Direction des médicaments vétérinaires.

En 2011, les antibiotiques de très haute importante en médecine humaine (catégorie 1) ont représenté respectivement $35 \%$ et $34 \%$ des achats totaux de DTQ/1 000 habitants-jour d'antibiotiques oraux et parentéraux (Tableaux 1 et 2). Ces pourcentages sont en hausse par rapport à 2001, année où les antibiotiques oraux et parentéraux avaient représenté respectivement $33 \%$ et $28 \%$ des achats.

La hausse de la consommation de médicaments parentéraux a été attribuable principalement à l'augmentation de la consommation des associations de pénicillines, incluant les inhibiteurs de $\beta$-lactamases (20\%), et des céphalosporines de troisième génération (14\%) (Tableau 2). 
TABLEAU 2. Nombre de doses thérapeutiques quotidiennes par 1000 habitants-jour d'antibiotiques parentéraux achetés par les hôpitaux au Canada, de 2001 à 2011

\begin{tabular}{|c|c|c|c|c|c|c|c|c|c|c|c|c|}
\hline & \multirow{2}{*}{ Classe ATC $^{1}$ et antimicrobien } & \multicolumn{11}{|c|}{ DTQ2/1000 habitants-jours } \\
\hline & & 2001 & 2002 & 2003 & 2004 & 2005 & 2006 & 2007 & 2008 & 2009 & 2010 & 2011 \\
\hline \multirow{7}{*}{$p^{p}$} & $\begin{array}{l}\text { Association de pénicillines, incluant les inhibite urs } \\
\text { de } \beta \text {-lactamases (J01CR) } \\
\text { Pipéracilline et inhibiteur enzymatique, ticarcilline et } \\
\text { inhibiteur enzymatique }\end{array}$ & 0,02 & 0,02 & 0,03 & 0,02 & 0,03 & 0,03 & 0,04 & 0,04 & 0,05 & 0,05 & 0,06 \\
\hline & $\begin{array}{l}\text { Céphalosporines de troisième génération (J01DD) } \\
\text { Céfotaxime, ceftazidime, ceftizoxime, ceftriaxone }\end{array}$ & 0,03 & 0,05 & 0,06 & 0,06 & 0,07 & 0,07 & 0,06 & 0,07 & 0,08 & 0,07 & 0,08 \\
\hline & $\begin{array}{l}\text { Céphalos porines de quatrième génération (J01DE) } \\
\text { Céfépime, ceftobiprole }\end{array}$ & $<0,01$ & $<0,01$ & 0,01 & 0,03 & 0,02 & 0,01 & 0,01 & $<0,01$ & $<0,01$ & $<0,01$ & $<0,01$ \\
\hline & $\begin{array}{l}\text { Carbapénèmes (J01DH) } \\
\text { Doripénème, ertapénème, imipénème, méropénème }\end{array}$ & 0,01 & 0,03 & 0,03 & 0,02 & 0,02 & 0,02 & 0,03 & 0,03 & 0,03 & 0,03 & 0,04 \\
\hline & $\begin{array}{l}\text { Fluoroquinolones (J01MA) } \\
\text { Ofloxacine, ciprofloxacine, norfloxacine, lévofloxacine, } \\
\text { moxifloxacine }\end{array}$ & 0,05 & 0,06 & 0,06 & 0,07 & 0,07 & 0,08 & 0,06 & 0,06 & 0,06 & 0,05 & 0,05 \\
\hline & $\begin{array}{l}\text { Glycope ptides (J01XA) } \\
\text { Vancomycine } \\
\end{array}$ & 0,04 & 0,04 & 0,02 & 0,02 & 0,02 & 0,01 & 0,01 & 0,01 & 0,01 & 0,02 & 0,02 \\
\hline & \begin{tabular}{|c|} 
Im idazole (J01XD) \\
Métronidazole
\end{tabular} & 0,06 & 0,02 & 0,01 & 0,01 & 0,01 & 0,01 & 0,01 & 0,01 & 0,01 & 0,01 & 0,01 \\
\hline \multirow{9}{*}{ \|I } & \begin{tabular}{|l|} 
Pénicillines à large spectre (J01CA) \\
Ampicilline, pivampicilline \\
\end{tabular} & 0,07 & 0,06 & 0,05 & 0,05 & 0,05 & 0,05 & 0,05 & 0,05 & 0,04 & 0,04 & 0,04 \\
\hline & $\begin{array}{l}\text { Pénicillines sensibles aux } \beta \text {-lactam ases (J01CE) } \\
\text { Pénicilline G }\end{array}$ & 0,21 & 0,19 & 0,22 & 0,27 & 0,28 & 0,28 & 0,26 & 0,27 & 0,26 & 0,25 & 0,25 \\
\hline & $\begin{array}{l}\text { Pénicillines résistantes aux } \beta \text {-lactamases (J01CF) } \\
\text { Cloxacilline }\end{array}$ & 0,05 & 0,04 & 0,05 & 0,04 & 0,04 & 0,04 & 0,04 & 0,04 & 0,05 & 0,04 & 0,04 \\
\hline & $\begin{array}{l}\text { Céphalosporines de première génération (J01DB) } \\
\text { Céfazoline, céphalothine }\end{array}$ & 0,12 & 0,12 & 0,12 & 0,12 & 0,12 & 0,11 & 0,12 & 0,12 & 0,13 & 0,12 & 0,12 \\
\hline & $\begin{array}{l}\text { Céphalosporines de deuxième génération (J01DC) } \\
\text { Céfotétan, céfoxitine, céfuroxime }\end{array}$ & 0,04 & 004 & 0 & ] & 0,1 & 001 & $001]$ & $0,01]$ & ก01 & $<001$ & $<0,01$ \\
\hline & $\begin{array}{l}\text { Macrolides (J01FA) } \\
\text { Azithromycine, érythromycine }\end{array}$ & 0,01 & 0,01 & 0,01 & 0,01 & 0,01 & 0,01 & 0,01 & 0,01 & 0,01 & 0,01 & 0,01 \\
\hline & \begin{tabular}{|l} 
Lincosamides (J01FF) \\
Clindamycine
\end{tabular} & 0,02 & 0,02 & 0,02 & 0,02 & 0,02 & 0,03 & 0,03 & 0,03 & 0,03 & 0,03 & 0,03 \\
\hline & $\begin{array}{l}\text { Am inoglucosides (J01GB) } \\
\text { Amikacine, gentamicine, nétilmicine, tobramycine }\end{array}$ & 0,06 & 0,05 & 0,03 & 0,02 & 0,03 & 0,05 & 0,05 & 0,04 & 0,04 & 0,04 & 0,03 \\
\hline & Total (J01) & 0,78 & 0,76 & 0,76 & 0,77 & 0,79 & 0,83 & 0,80 & 0,79 & 0,82 & 0,77 & 0,79 \\
\hline
\end{tabular}

${ }^{1} \mathrm{ATC}=$ Système de classification anatomique, thérapeutique et chimique

${ }^{2} \mathrm{DTQ}=$ Dose thérapeutique quotidienne

${ }^{3}$ Les chiffres romains I et II indiquent l'importance des antibiotiques en médecine humaine, selon les catégories du système de classification établi par la Direction des médicaments vétérinaires.

Les fluoroquinolones représentent le groupe d'antibiotiques oraux le plus consommé (24 \%). Viennent ensuite les macrolides (15\%), les tétracyclines (12\%), les céphalosporines (12\%) et les pénicillines à large spectre (11\%) (Figure 1). 
FIGURE 1. Pourcentages du nombre total de doses thérapeutiques quotidiennes d'antibiotiques oraux achetées par 1000 habitants-jour par les hôpitaux au Canada, 2011

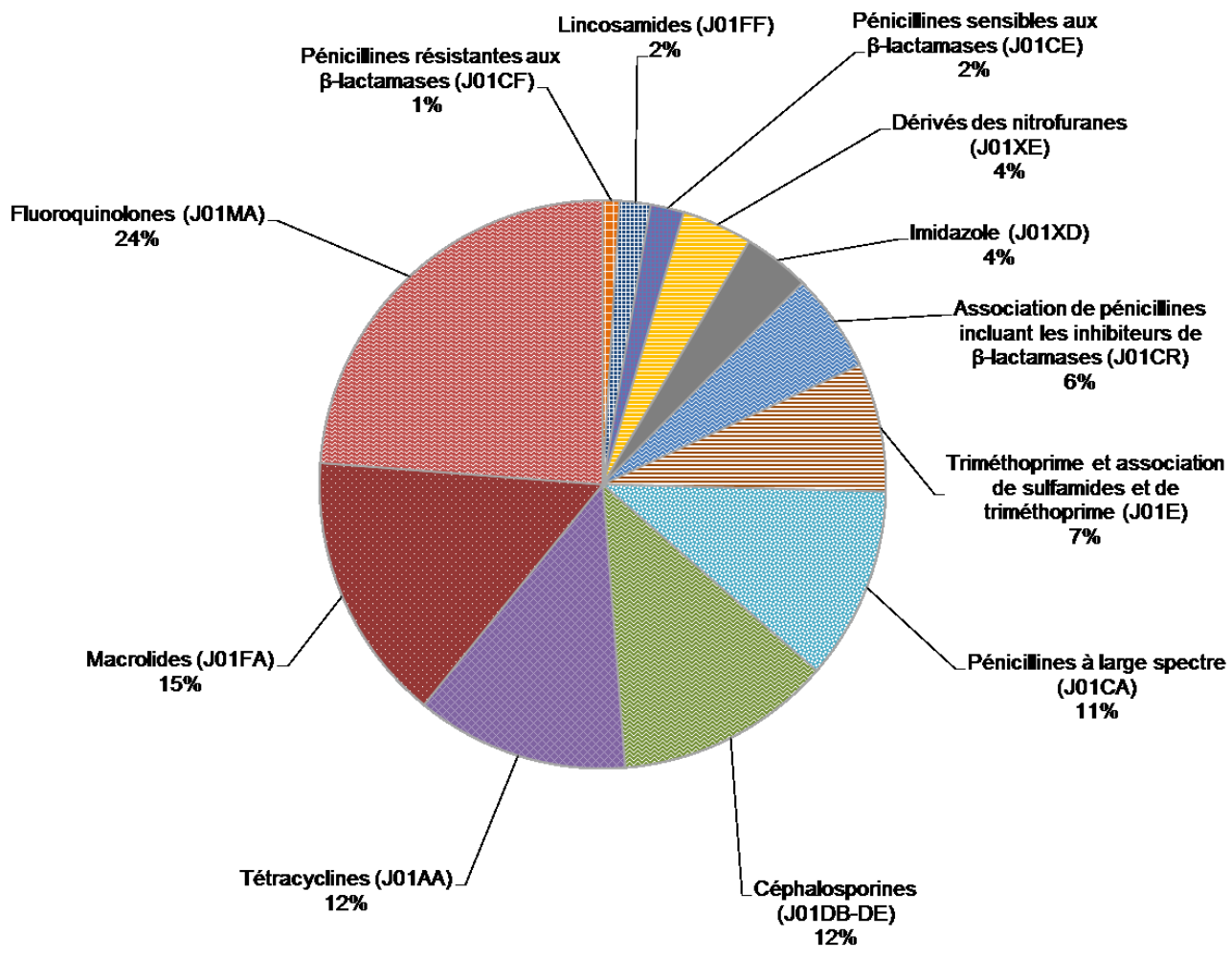

Note : Les codes alphanumériques indiqués entre parenthèses sont ceux du Système de classification anatomique, thérapeutique et chimique des antibiotiques.

Les pénicillines sensibles aux $\beta$-lactamases (pénicilline $\mathrm{G}$ et pénicilline $\mathrm{V}$ ) (32 \%) et les céphalosporines $(26 \%)$ représentent les plus importants groupes d'antibiotiques parentéraux en termes d'achats par les hôpitaux canadiens (Figure 2). 
FIGURE 2. Pourcentages du nombre total de doses thérapeutiques quotidiennes d'antibiotiques parentéraux achetées par 1000 habitants-jour par les hôpitaux au Canada, 2011

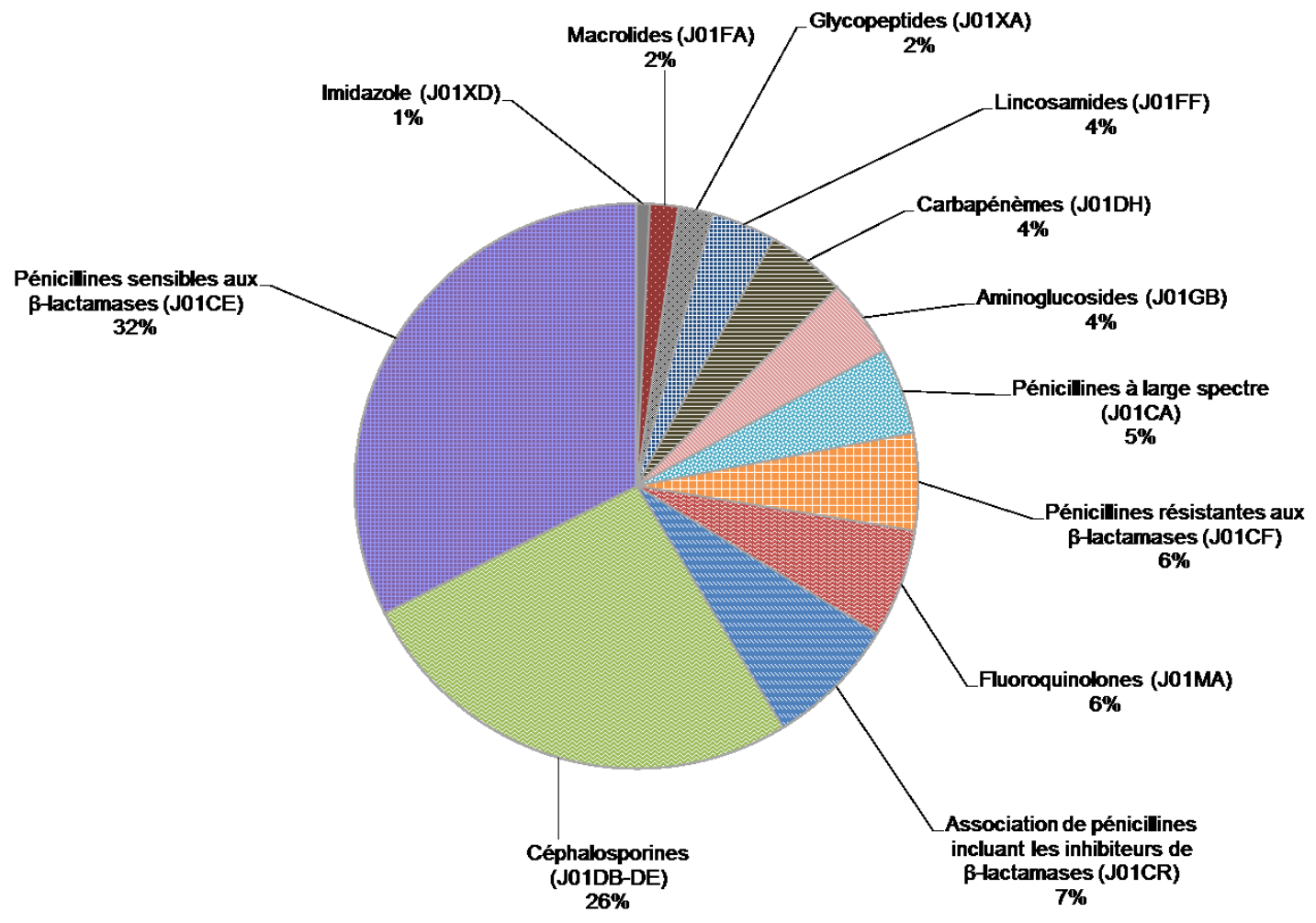

Note : Les codes alphanumériques indiqués entre parenthèses sont ceux du Système de classification anatomique, thérapeutique et chimique des antibiotiques.

En 2011, des différences ont été observées d'une province à l'autre sur le plan de la consommation totale d'antibiotiques et du coût total en dollars (par 1000 habitants-jour) des antibiotiques achetés par les hôpitaux canadiens. Dans l'ensemble, c'est au Manitoba que la consommation d'antibiotiques a été la plus forte (2,61 DTQ/1 000 habitants-jour) et en Ontario qu'elle a été la plus faible

(1,26 DTQ/1 000 habitants-jour) (Figure 3). En ce qui concerne le coût total des antimicrobiens achetés par les hôpitaux, c'est en Colombie-Britannique qu'il a été le plus élevé (12,13 \$/1 000 habitants-jour) et en Ontario qu'il a été le plus bas (6,72 \$/1 000 habitants-jour) (Figure 3). 
FIGURE 3. Nombre de DTQ/1 000 habitants-jour (consommation totale) et coût total des antibiotiques oraux et parentéraux achetés par les hôpitaux des provinces canadiennes, 2011

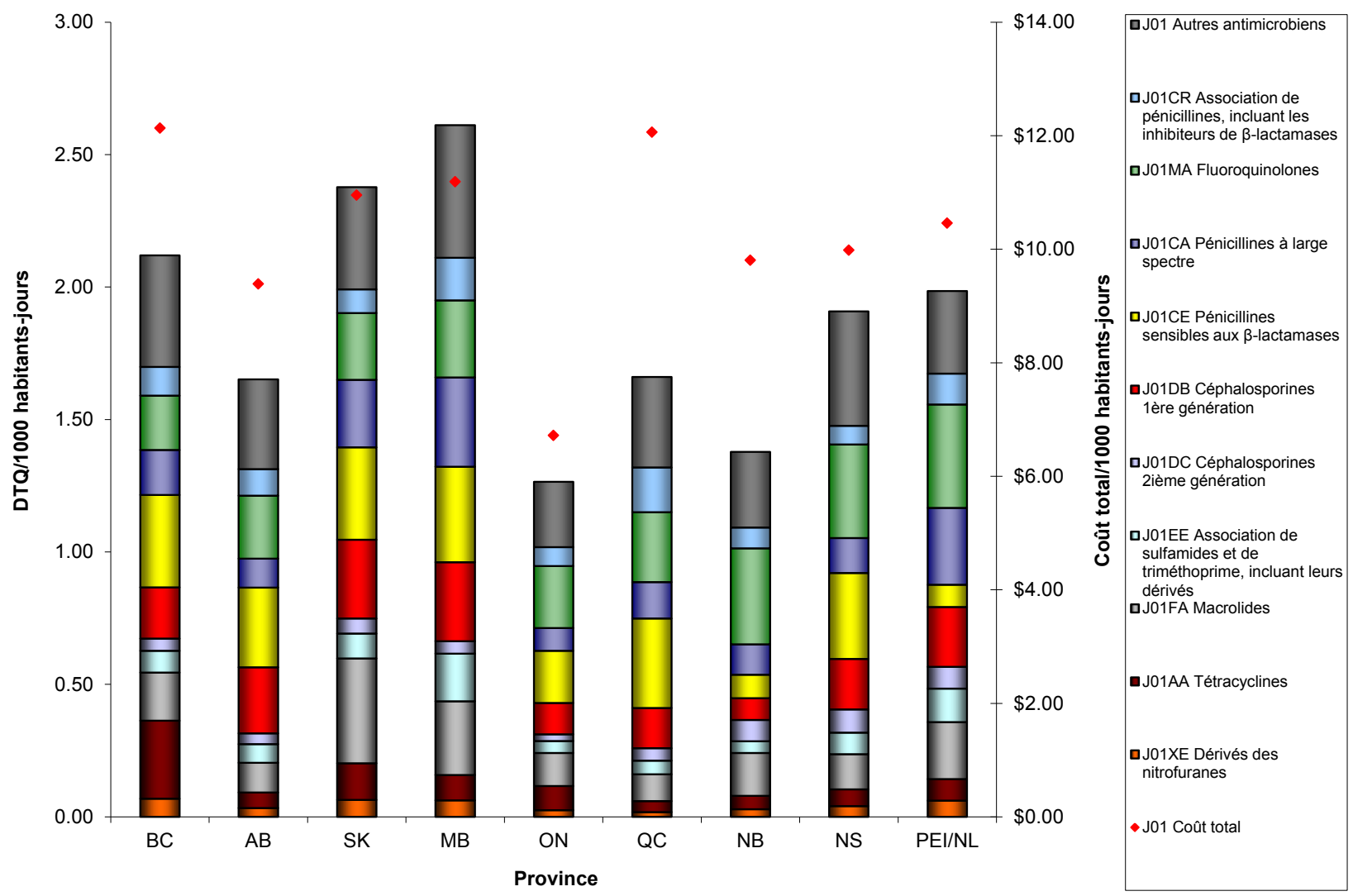

Note : Les codes alphanumériques indiqués entre parenthèses sont ceux du Système de classification anatomique, thérapeutique et chimique des antibiotiques.

\section{Discussion}

De 2001 à 2011, les achats totaux d'antibiotiques par les hôpitaux canadiens ont augmenté de $27 \%$ $(9448,05 \mathrm{~kg})$, de légères augmentations ayant été observées dans la consommation d'antibiotiques oraux (hausse de $8 \%$ ) et parentéraux (hausse de $3 \%$ ). En 2011, les antibiotiques considérés comme étant de très haute importance en médecine humaine (catégorie I) ont continué de représenter un pourcentage élevé $(0,56 / 1,62 \mathrm{DTQ} / 1000$ habitants-jour) de la totalité des antibiotiques utilisés dans les hôpitaux.

Dans l'ensemble, c'est au Manitoba que la consommation d'antibiotiques (DTQ/1 000 habitants-jour) a été la plus forte (2,61 DTQ/1 000 habitants-jour) et en Colombie-Britannique que le coût total en dollars (par 1000 habitants-jour) a été le plus élevé (12,13 \$ par 1000 habitants-jour). À l'opposé, I'Ontario a affiché la consommation la plus faible (1,26 DTQ/1 000 habitants-jour) et le coût global le plus bas $(6,72 \$$ par 1000 habitants-jour). En 2011, la plus forte consommation d'antibiotiques de catégorie I (43\%; 0,62/1,43 DTQ/1 000 habitants-jour) a été enregistrée au Nouveau-Brunswick, un record attribuable à l'augmentation de la consommation de fluoroquinolones dans cette province.

Il convient de préciser que l'ensemble de données du Canadian Drugstore and Hospital Purchases Audit comporte plusieurs limites et restrictions. Ainsi, les données sont des estimations et non des données de recensement; de plus, certains marchés à créneaux précis font l'objet d'un suivi limité (en raison du faible 
volume ou d'une distribution unique). II est possible également qu'un petit nombre de produits aient été exclus pour des raisons de confidentialité (p. ex. s'ils ne sont vendus que dans un seul point de vente). Certaines données peuvent aussi être exclues pour refléter les tendances réelles du marché (p. ex. transactions visant à constituer de vastes réserves en prévision d'une épidémie potentielle), et les ventes directes d'un fabricant en particulier peuvent être manquantes, ceci donnant lieu à une sous-estimation des achats d'un produit (incidence plus élevée dans le cas des achats par les hôpitaux que de ceux effectués par les pharmacies).

Les provinces de l'Île-du-Prince-Édouard et de Terre-Neuve-et-Labrador ont été regroupées en raison du faible volume d'achats dans chacune de ces provinces. Les changements apportés aux bases de données ne portent que sur les 72 derniers mois et, au-delà de six ans, les ensembles de données sont considérés comme fermés, car aucune mise à jour n'est possible. Comme le nombre de jours d'hospitalisation et de lits d'hôpitaux n'était pas disponible pour ces données, les renseignements sur la population générale ont été utilisés pour produire les taux d'achat.

La résistance aux antimicrobiens continuera à mettre en danger la santé des Canadiens et des autres habitants de la planète pendant encore un certain temps. La surveillance de l'utilisation des antibiotiques aidera à documenter les progrès que nous continuerons de faire pour freiner cette tendance.

\section{Remerciements}

L'auteur aimerait souligner la contribution des réviseurs externes du Rapport sur l'utilisation des antimicrobiens chez les humains : $D^{r}$ John Conly, $D^{r}$ Jim Hutchinson, $D^{r}$ Warren Mclsaac, $D^{r}$ David Patrick, $D^{r e}$ Lynora Saxinger, $D^{r}$ Daniel Thirion et $D^{r}$ Karl Weiss. Sans leur étroite collaboration et leur participation, l'analyse et la présentation de ces données n'auraient pas été possibles.

\section{Conflit d'intérêts}

II n'y a aucun conflit d'intérêts à déclarer.

\section{Financement}

Ce travail a été appuyé par l'Agence de la santé publique du Canada.

\section{Avertissement}

Cet article a été préparé grâce aux données d'IMS Health Canada Inc. Les analyses, les conclusions, les opinions ainsi que les énoncés exprimés dans cet article sont ceux de l'auteur et non ceux d'IMS Health Canada Inc.

\section{Références}

(1) Agence de la santé publique du Canada. Programme intégré canadien de surveillance de la résistance aux antimicrobiens (PICRA), Rapport sommaire sur la résistance aux antimicrobiens, 2011. Guelph (Ontario), Agence de la santé publique du Canada, 2012. http://publications.gc.ca/collections/collection_2013/aspcphac/HP2-4-2-2011-fra.pdf 